Mr tratter Itrugh.

Divicion of Ethnology U.S. National Musoum

\title{
ORNITHOLOGICAL VOCABULARY
}

\author{
Uivislon of Ethnology
}

U.S. National Museum

OF THE

677

.M477

1896

ANTH

\section{MOKI INDIANS}

$\mathrm{BY}$

DR EDGAR A. MEARNS, U.S. A.

[From The American Anthropologist for December 1896]

WASHINGTON, D. C.

JUDI \& DETWEILER, PRINTERS

1896 



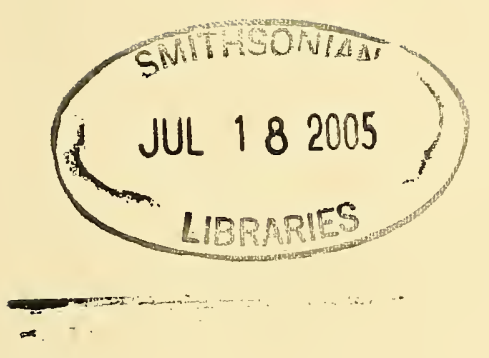




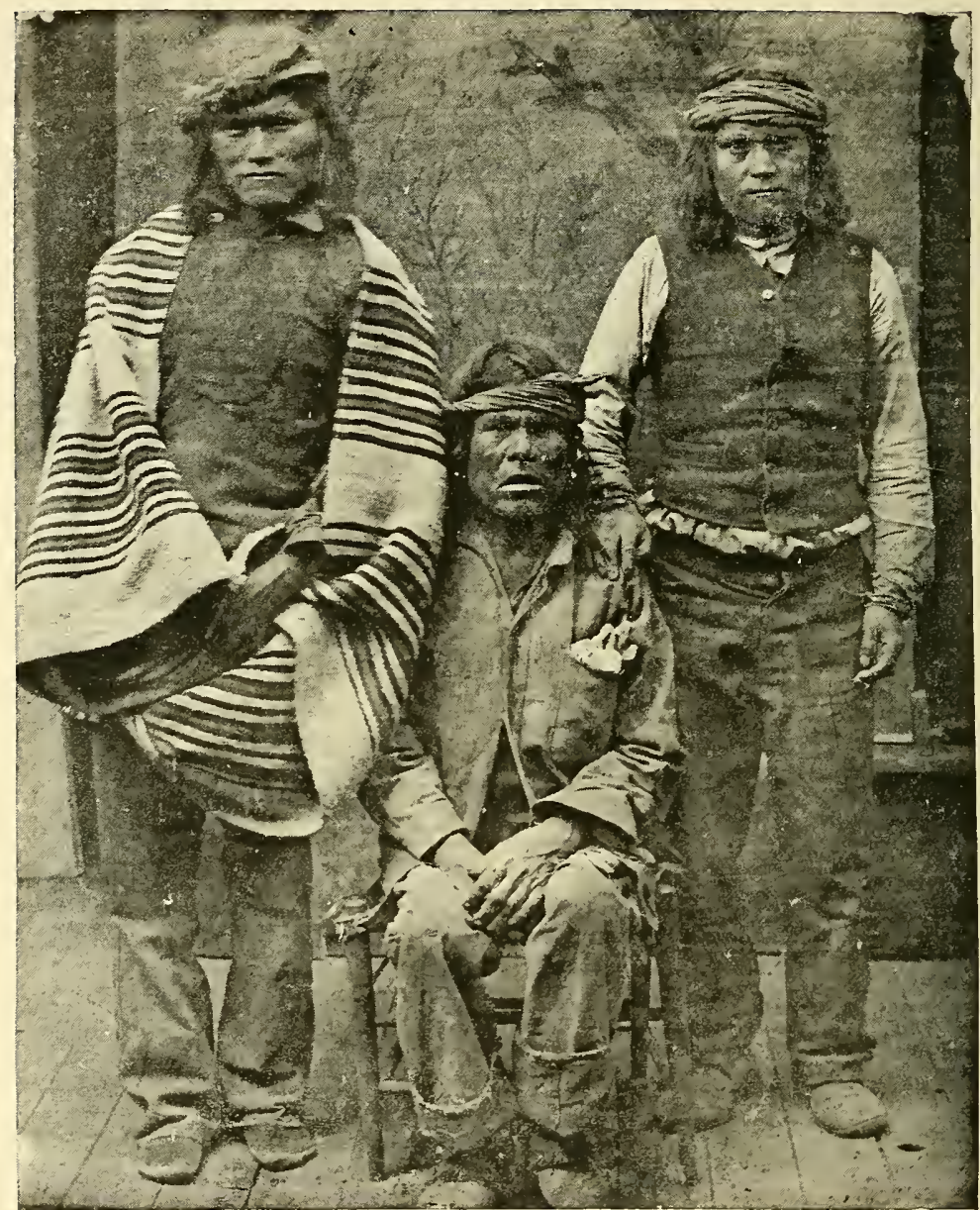




\section{ORNITHOLOGICAL VOCABULARY OF THE MOKI INDIANS}

DR EDGAR A. MEARNS, U. S. A.

The people whose ornithology I have attempted to portray are a tribe of more than ordinary interest, whether judged from an ornithologist's point of view or otherwise. The Mokis inhabit a region of country in longitude $109^{\circ}$, lying just west of the New Mexico-Arizona boundary, nor theastward from Little Colorado river, and 65 miles south of the Colorado. Their seven villages or pueblos are Oraibi, Shumopavi, Shipaulovi, Mashongnavi, Walpi, Sichumovi, and Hano or Tewa. Each is built on the lofty crest of a precipice of sandstone, and combines a town and fortress which is impregnable to any assault to be anticipated from aboriginal foes. Thus romantically situated are the seven Moki cities today, although they do not occupy the sites of the villages first seen by the Spaniards, who were the first Europeans whose feet pressed the soil of Arizona in the early part of the sixteenth century. The people were then peaceful, intelligent, and industrious; they raised good crops of corn, beans, and pumpkins, wore cotton cloth and dressed deerskins, and were in no respect materially different from their descendants of the present day. Aside from these olden records, the Mokis have a far more ancient history, written in monuments scattered over their present domain and probably as far southward as the Gila river. These monuments are the remains of single-housc structures as well as towns, built of stone and adobe, many of them wonders of aboriginal architecture. Some of these structures are cliff or cave dwellings, whose builders were the immediate ancestors of the Mokis and Zunis, as these In- 
dians themselves assert; and indeed there is little ground for doubting this, although much mystery has been made to surround these ruined edifices, whose origin has been variously accredited to Aztecs and other races. Many human skeletons which I have exhumed from the burial cists of cliff-dwellings bear an almost exact resemblance to the form and stature of the Mokis, and there is a close correspondence between the implements and materials found buried in the caves and casas grandes of the extinct people and those now in use or still preserved as relics in the houses of the Mokis. All of these proofs declare that the Mokis were once a more numerous people than now, and that they have long been in possession of the country they today occupy.

The Mokis are a thrifty race, devoted to agricultural pursuits and to raising sheep, goats, cattle, ponies, donkeys, hogs, and some turkeys and chickens. These and other occupations keep them employed at home during most of the year; but in autumn or winter they make frequent visits to the neighboring Indian tribes-the Havasupais, Navahos, and Apaches, as well as the Zuñis and other Pueblo tribes. Before its abandonment, they visited Fort Verde at least once a year, the trip occupying six days, as they travel, riding ponies and packing burros. Sometimes they extend the journey forty miles for the purpose of trading at Prescott. They formerly made occasional pilgrimages across the parched desert country to the Pacific ocean, and returned laden with seashells and other products.

The revision of the zoölogical vocabulary of the Moki language, of which the present paper forms the ornithological portion, was made with the aid of my venerable friend Ongwischey (Raven), who fully comprehended my motives and exerted himself to make the list of names as complete and accurate as possible. Ongwischey is an exceedingly intelligent Indian, with many commendable qualities; he is simple and truthful, speaks fairly good English, and hence proved invaluable. He also possesses an excellent knowledge of the fauna, having traveled extensively in Arizona and New Mexico, but he has never been a hunter, although his brother Näh'hŭh (Duck), chief or governor of one of their villages, is a Nimrod of the Moki tribe, having killed many mountain sheep, deer, antelope, and other large game; but the acme of the „Moki hunter's aspirations was 
achieved when he killed an elk in the White mountains of Arizona, an exploit which his people do not intend shall be forgotten. Some hunters among the young men were found to have a better acquaintance with the pinicoline species, with which they met when hunting in the high mountains, and Ongwischey acted as interpreter for them.

My contact with the Moki Indians has been for such brief periods and so intermittent that I have by no means mastered their language or even its grammatical construction, although it appears to resemble the English in the form of its descriptive names, with which the present paper has exclusively to do.

It will be observed that some of the Moki names are of Spanish origin. The fact is the Moki tongue has become impure from contact with Mexicans and half-bloods from some of the New Mexican pueblos, where Indians and Mexicans live together.

Although more attentive to nature than most whites, it must be remembered that the Mokis are not ornithologists, and cannot be expected to name even all birds that have fallen under their observation, much less such as have never attracted their critical attention, or to discriminate between closely related species or those which resemble one another in color or form. In the accompanying list I have included all species of Arizona birds for which I could obtain any name, although some of them were manifestly coined at the moment. The bird was invariably held in hand, or, whenever possible, shown to them alive, at the time its Moki appellation was transcribed. Perhaps when some small sparrow was presented for christening they would call it simply $c^{-} \bar{e}^{-}-\bar{e}$ (little bird), and, when closely pressed for a more distinctive title, they would call it pŭn'tō-chēe- (spotted little bird). It was thought worth while to record names thus obtained, if for no other purpose than to show the limitations of their ornithological knowledge. I have written the Moki names phonetically, as pronounced by the Indians, and have not hesitated to vary the spelling to suit variations in pronunciation by different individuals, thinking thereby to make this vocabulary more useful and intelligible to those who may subsequently acquire a mastery of the language and desire to construct a complete Moki vocabulary.

The Mokis have a superstitious regard for most living things, and many animals are held by them as sacred and made the 
object of idolatrous worship. Ophiolatry is practiced by them; and so great is their reverence for serpents that they will not permit one to be molested. They can name any indigenous serpent without the slightest hesitancy, thus showing their great familiarity with them. The revolting religious rites practiced by them at the time of their annual Snake-dance are fully described by Captain John G. Bourke, U. S. A., ${ }^{1}$ and by Dr J. Walter Fewkes. $^{2} \quad$ Among the birds held sacred, or which represent their clans or secret religious orders, are the eagle, parrot, macaw, heron or sandhill crane, road-runner or chapparal cock, turkey, and dove. Observers of Moki ceremonies have seen large wooden tablets in their kivas or ceremonial chambers painted with a green ground, ornamented with the rain prayer and some one of the countless Moki gods, and have remarked that the little bird in the clouds suggests the Thunder-bird of the Plains Indians. Bourke states that "feathers appear constantly in religious ceremonies"-chiefly those of the eagle and turkey. 'The wands, made with eagle feathers, used for fanning living serpents at their Snake-dance, cannot be bought, as they refuse to part with them for any pecuniary consideration, fearing to offend their ornithological deity. "In the early days of spring, when the fields are to be tilled, the devout Mokis prepare the sacrificial plumes of eagle down attached to little sticks, which are buried in the corners of their lands." 3 Bancroft, speaking of the natives of Mexico, tells us (vol. II, p. 234) that eagles were furnished as tribute to Montezuma, one town alone sending in forty each year. He also informs us that a species of paroquet was a sacred bird among the Zapotecs of Mexico, and as such worshiped (vol. II, p. 211). Bourke observes: "The feathers of the parrot, which have to be brought up from the interior of the neighboring Republic of Mexico, are treasured by all the Pueblos as far north as Taos and Picuris, and west to Acoma, Zuñi, and Oraibi. They will always be found carefully preserved in peculiar wooden boxes, generally cylindrical in shape, made expressly for the purpose; with them is invariably associated the soft white down of the eagle." He also notes having seen caged parrots in the pueblo of Santo Domingo, New Mexico, which

1 The Snalie-dance of the Moquis of Arizona, ete, New York, 1884,

2 Snake Ceremonials at Walpi : Jour. Am. Eth. and Arch., vol. Iv, Boston, 1894.

3 Bourke: Snake-dance of the Moquis, p. 259. 
were in a fearful state of demoralization, nearly every one of their fine blue, red, and yellow feathers having bcen plucked to make wands for the dancers or to decorate the sacred standards used in their Dance of the Tablet.

The Mokis show an excellent acquaintance with raptorial birds. They have an especial veneration for the two species of eagle, which are kept by them in cages in the pueblos of Oraibi, Mashongnavi, Shumopavi, and Shipaulovi, ${ }^{1}$ and fed largely on field-mice and rabbits. Captain Bourke alludes to eagle feathers as common articles of commerce among the Mokis, having a determinate value, and ascribes the high pricc placed upon them by all the sedentary Indians of Arizona and New Mexico to some considerations graver than those of commercc.

\section{Vocabulary}

\section{General Terms}

Bird, qua-yŭh'

A big bird, qua'hŭh.

A little bird, çhè'é.

A bird's nest, $t \ddot{t} h^{\prime} k \bar{e}$. When shown a nest of the black phobe (Sayornis nigricans), they called it a wowwow'ŭh-k'ic-täh'ke, meaning a swallow's nest, which it resembles.

A bird's egg, qua'nŭ-hŭh.

To sing, tûr-tŭck' $\breve{u} h$.

To fly, phûr-yăct'te.

To run, härzh-rŏŏor'ru-tă.

Nan, täh'kă.

Woman, wurk'te.

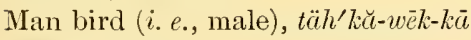
or $t \ddot{a} h^{\prime} k \breve{a}-c ̧ h \bar{e}^{\prime} \bar{e}$.

Woman bird (i. e., female), wurk'tewēk-kì or wûrk'tēeşhè-e.

Old, wârk'tŏk-äh.

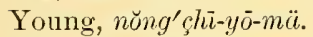

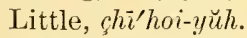

Big, yä $h^{\prime} s ̧ \bar{\imath}-y \breve{o r} k-k \breve{a}$.

Water, päh'hŭh or kûr'ye.

River, çhûr-hûrbp'bè-coil-lǔ̆h.

Mountain, nē-wüt'te- -kow-ê.
Stone, öh-hua' or ōh-wvŭh'

Tree, schè-hûrbp'bè.

Cottonwood-tree, päh-schēe-hû́bp'bē.

The ground or earth, whär'zhü.

Grass, püh-tish'n'wü.

Spotted, p̌n'tōo (Spanish).

Black, coim'bwē.

White, coy-ōte'să.

Green, mō-kĭng'pŭh.

Blue, shais-quä-bŭh.

Yellow, tŏsh'căb-by.

Red, päh'lä.

Brown, tơsh-ēelè.

Head, coit-tûr-ăt-tă.

Shoulder, sçȟ̆l'kă-ăt-tă.

Wing, müh'schŭ-ăt-tŭ.

Tail, sçhä-rōō' $\breve{t} t-t \breve{a}$.

Leg, hō-käh' ăt-tŭ.

Foot, quir $k^{\prime}$ ŭt-ť̆.

Eye, $p \bar{o}^{\prime} s c ̧ h e-\breve{a} t-t \breve{u}$.

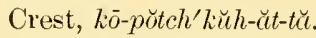

Little bill or beak, mö-çhōp'hō-yŭh.

Big bill or beak, will-kōm'n'chō-wŭh. Gun, äh-mûrk'̌̆̌h-bēe.

1 Captain Bourke states (p. 27) that "eagles are still raised in cages in Picuris, San Ildefonso, Santa Clara, Zuñi, Acoma, and the villages of the Moquis farthest to the west." 
Specific Terms

Podilymbus podiceps (Linn.), pied-billed grebe, sçhirp'kiēn-äh.

Urinator imber (Gunn.), loon, çhä-cum'ŏn. (A "swift diver.")

Urinator pacificus (Lawr.), Pacific loon, ç九̈̈-cum'ŏn.

Larus dela warensis Ord., ring-billed gull, päh'qua-řh. (A “fisherman.")

Sterna forsteri Nutt., Forster's tern, päh-wōke'kō.

Hydrochelidon nigra surinamensis (Gmel.), black tern, coim'bwē-pähwōke'ko.

Merganser americanus (Cass.), A merican merganser, tŏng' äh-coit-r̆h .

Nerganser serrator (Linn.), red-breasted merganser, $h \bar{o}-p \breve{t} t c h^{\prime} k \bar{o}-\imath v o w-\bar{e}-k \breve{a}$.

Lophodytes cucullatus (Linn.), hooded merganser, cō-çhä'he--qua-wowwēk-kă.

Anas boschas Linn., mallard, tŏng-ä $h^{\prime}$ cow-wow-ŭh-kie.

Anas strepera Linn., gadwall, päh'week-ěh' or päh'wé-k'ia.

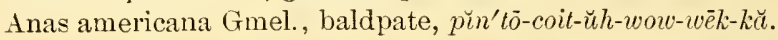

Anas carolinensis Gmel., green-winged teal, çhō-cōne'wow-wēk-kă.

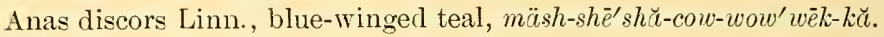

Anas cyanoptera Vieill., cinnamon teal, päh'wè-k'ia.

Spatula clypeata (Linn.), shoveller, päh'wē-k'ia.

Dafila acuta (Linn.), pintail, p̆n'tō-wow-wēk-kă.

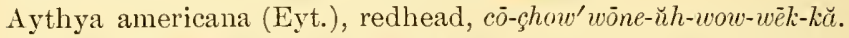

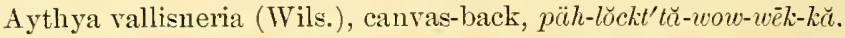

Aythya collaris (Donov.), ring-necked duck, pāy'tŭ-cow-wow-wēk-kă. (Refers to the band across its bill.)

Charitonetta albeola (Linn.), buffle-head, wŭsçh-éewow-wēk-ǩ̆.

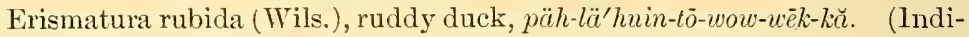
cates a duck spotted with red.)

Branta canadensis occidentalis (Baird), white-cheeked goose, pûhr-

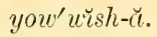

Branta canadensis minima Ridgw., cackling goose, w̌̆ll-kō'wow-wēk-kău.

Olor buccinator (Rich.), trumpeter swan, päh'to-kio.

Note то Anatidx. - Päh-wow-wow'ict-tá. A general name used by the Mokis to denote any member of this family. Mokis do not eat ducks, but the name (näh'h $\breve{\iota} h$ ) of the chief or governor (called moung'we) of a village $(h \bar{o}-p \bar{e} \cdot k \bar{e})$ signifies a species of duck.

Plegadis guarauna (Linn.), white-faced glossy ibis, päh-lä'tông-ŭh-powwow'-wè k-kŭ.

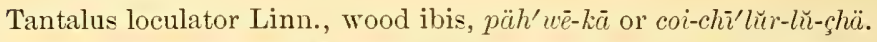

Botaurus lentiginosus (Montag.), American bittern, w̌ll-kö'huatch-rŏ̌̆.

Ardetta exilis (Gmel.), least bittern, cō-şh $\ddot{a}^{\prime} h \bar{e}-q u \alpha$.

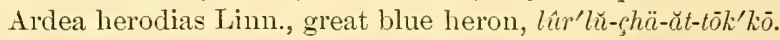

Ardea egretta Gmel., American egret, cō-çhow'wou-wēk-k̆̆.

Ardea candidissima Gmel., snowy heron, cō-çhow'he-me--wow-wēel-kă.

Ardea rufescens Gmel., reddish egret, $p \ddot{a} h-l \ddot{a}^{\prime} \breve{a} t-t \bar{o} k^{\prime} k \bar{o}$.

Ardea virescens anthonyi Mearns, green heron, mŭsh-shēéwow-ē-kă.

Nycticorax nycticorax nævius (Bodd.), black-crowned night heron, cō-

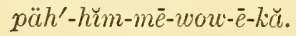


Grus mexicana (Müll.), sandhill crane, wüll-päh'qua-pbè.

Rallus virginianus Linn., Virginia rail, coim’ŭp-huatch-rŏ̌.

Porzana carolina (Linn.), sora, päh-lä'huatch-rŏð.

Gallinula galeata (Licht.), Florida gallinule, päh-lä'çhē-kum'n-äh

Fulica americana Gmel., American coot, çhē-kum'n'äh-nu-mŭsh-tŏł-ï.

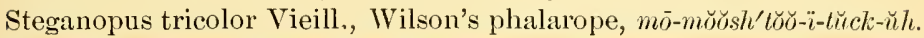

Recurvirostra americana Gmel., American avocet, w̌̆ll-pŏm'çhō-wŭh.

Gallinago delicata (Ord.), Wilson's snipe, päht-huat'ēeōo-shōne-ěh .

Tringa bairdii (Coues), Baird's sandpiper, päh'çhē-rŏŏ.

Tringa minutilla Vieill, least sandpiper, pŏtch'rŏ̌̆-hōe-y̆ŭh.

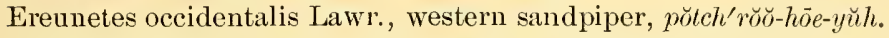

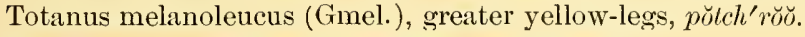

Totanus flavipes (Gmel.), yellow-legs, pŏtch-rŏŏ.

Totanus solitarius cinnamomeus Brewster, western solitary sandpiper, cō-çhäh' 'huatch-rŏŏ.

Symphemia semipalmata inornata Brewster, western willet, cö-çhäsh'zhrū.

Actitis macularia (Linn.), spotted sandpiper, mäch-shē'huatch-rŏø̆.

Numenius longirostris Wils., long-billed curiew, will-cō'luü̈-cāle-lè.

Egialitis vocifera (Linn.), killdeer, päh'kāle-lè.

Egialitis montana (Towns.), mountain plover, päh'käle-höe-yŭh.

Gallus gallus (Linn.), domestic fowl, $c u-a ̈ h^{\prime} c u$. (The Mokis raise a small variety of fowl, a little larger than the bantam, which they keep in stone houses built for the purpose.)

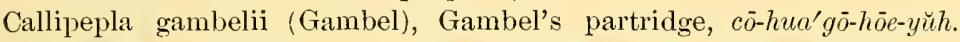
When asked to name a specimen of the scaled partridge (Callipepla squamata), they replied, "No sabe ; no got 'em Moki country."

Cyrtonyx montezumæ (Vig.), Nlassena partridge, $p^{\prime} n^{\prime} t \bar{o}-h \bar{o}-i a$.

Meleagris gallopavo mexicana (Gould), Mexican turkey, coi-yung'ō. The tuft of coarse hair growing from its breast they call coi-yung-work-sē. This bird was domesticated, history informs us, early in the sixteenth century, at the time of the early Spanish invasion, not only by the Mokis, but by other sedentary Indian tribes in New Mexico and Arizona. ${ }^{1}$ I have frequently found turkey bones when excavating in the ruins of the cliff and cave dwellers in the valleys of the Rio Verde and Salinas.

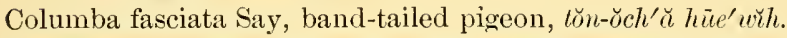

Zenaidura macroura (Linn.), mourning dove, päh-lä' hĭe-v̌̌h.

Melopelia leucoptera (Linn.), white-winged dove, pin'tō hüe-w̌̌h.

Columbigallina passerina pallescens (Baird), Mexican ground dove, pählä'hūe-w̌̌h .

Scardafella inca (Less.), Inca dove, hïe'e- è̌ wh-qua.

Cathartes aura (Linn.), turkey vulture, wē-shōke'ke.

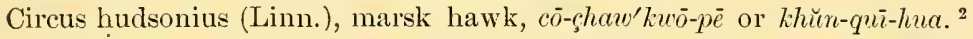

1 In this connection see Davis's Conquest of New Mexico. The turkey was also domesticated by the Aztecs. See Brancroft's Native Races of the Pacific States, II, 353, I875.

2 Qua-yŭh or qua-jă is their generie term for hawks, and upon the word they ring various changes. 
Accipiter velox (Wils.), sharp-shinned hawk, cō-çhaw' hung'wē-ǩ̆. Accipiter cooperii (Bonap.), Cooper's hawk, k'e'säh or hung-hü̈-kă. Accipiter atricapillus (Wils.), American goshawk, p'te'quйn-quäy-lıua. Parabuteo unicinctus harrisi(Aud.), Harris's hawk, pähl-lä'poit-gŭ-quāy-wă.

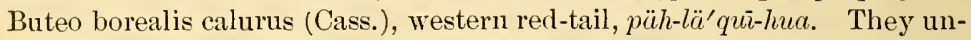
derstand the various ageal and irregular phases of plumage and apply the same term-päl'l$l \ddot{a}$ (red)-to all specimens of this species.

Buteo abbreviatus Caban., zone-tailed hawk, $h \bar{o}^{\prime} q u \bar{\imath}-h u a$ or $h \bar{o}^{\prime} q u \bar{\imath}-y \breve{u} h$.

Buteo swainsoni Bonap., Swainson's hawk, quēequè'quī-yō.

Urubitinga anthracina (Licht.), Mexican black hawk, qua'hüe-yй-yŭh.

Aquila chrysaëtos (Linn.), golden eagle, tzlll-whōōsh'hēap-qua-hûr.

Haliæetus leucocephalus (Linn.), bald eagle, né-huack'qua-hûr.

Falco mexicanus Schleg., prairie falcon, $k \bar{e}^{\prime} s \ddot{\prime a ̈ h}$.

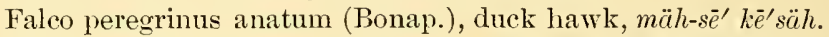

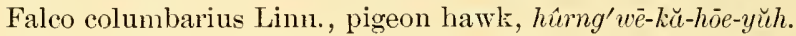

Falco sparverius deserticolus Mearns, desert sparrow hawk, täh-lkä́cāle-lēe

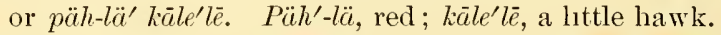

Pundion haliaëtus carolinensis (Gmel.), American osprey, coy-ç hŭckt'të. The name refers to the white on its head.

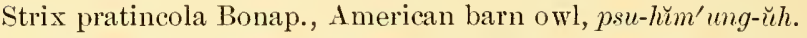

Asio wilsonianus (Less.), American long-eared owl, psu-hı̆m'ung-ц̌h.

Megascops asio cineraceus Ridgw., Mexican screech owl, ş̧hĭ-lăp'mŏðnghua.

Bubo virginianus occidentalis Stone, western horned owl, mŏðng'hua or mŏŏng'wä.

Speotyto cunicularia hypogæa (Bonap.), burrowing owl, $k \bar{o} k_{\bar{o}} \bar{o}$ or pä $h^{\prime} l \breve{u}_{-}$ mŏong' üh.

Glancidium gnoma Wagl., pygmy owl, köe'rhän-e-e The ferruginous pygmy owl (Glaucidium phalænoides) is regarded by the Mokis as identical with this species.

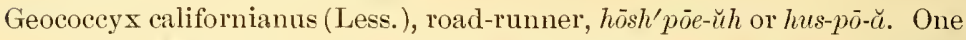
of the Mokis' clans or gentes is named after the road-runner. Ongwischey belongs to this clan.

Coceyzus americanus occidentalis Ridgw., California cuckoo, ko' $k \bar{o}$ hǚe' w̌̌h or cō-çhow'huin-ō hiee-w̌h. They class the cuckoo with the pigeons.

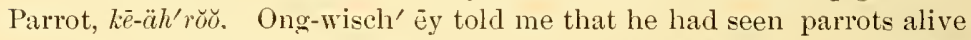
only at the pueblos on the Rio Grande, in New Mexico. The Mokis use parrot feathers in their religious ceremonies with the sacred eagle feathers, and regard the bird with veneration. Ongwischey was delighted with a pasteboard representation of one of these birds, which was presented to my little child for a plaything, and carried it back to the Moki villages with him.

Ceryle alcyon (Linn.), belted kingfisher, çhē-kum'n'äh, a name applied in common to several water-loring birds.

Dryobates villosus hyloscopus (Cab.), Cabanis's woodpecker, höte'päh-kŭ coy-ōte'sŭ or pŭn'tō höpe'yung-äh. 
Dryobates pubescens homor'us (Cab.), Batchelder's woodpecker, hōpe'yung-äh.

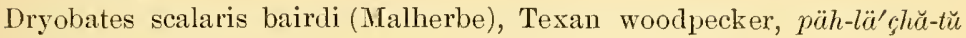
hōpe-yung-äh.

Sphyrapicus varius nuchalis Baird, red-naped sapsucker, päh-la'hē-quä hōpe'yung-äh.

Sphyrapicus ruber (Gmel.), red-breasted sapsucker, päh-løcht'tē hōpe'yung-äh.

Sphyrapicus thyroideus (Cass.), Williamson's sapsucker, hōpe'yung-äh.

Melanerpes formicivorus Swainson, Mexican woodpecker, coim'bwè hōpe'yung-äh.

Melanerpes torquatus (Wils.), Lewis's woodpecker, coim'bwē (black) mŭzy̆ng'ow-ц̌̆h (flicker).

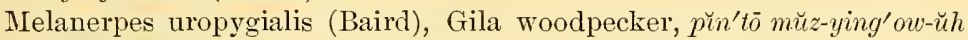
(spotted flicker).

Colaptes cafer (Gmel.), red-shafted flicker, mŭz-ying'ow-ŭh.

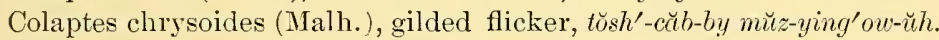

Phalænoptilus nuttallii (Aud.), poor-will, hōe-ütsk-gō (= accent).

Chordeiles acutipennis texensis (Lawr.), Texan nighthawk, you' $\bar{e}-b \bar{e}-q u a^{\prime}$.

Aëronautes melanoleucus (Baird), white-throated swift, ōh-mow'wow-wowǔh-k'ia.

Trochilus alexandri (Bourc. \& Muls.), black-chinned hummingbird, tōke'ş九̆.

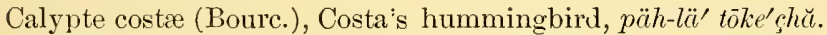

Selasphorus platycercus (Swains.), broad-tailed hummingbird, tōke'çhă.

Selasphorus rufus (Gmel.), rufous hummingbird, päh-lä' tōke'çhă.

Iache latirostris (Swains.), broad-billed hummingbird, tōke'çhă.

Tyrannus verticalis Say., Arkansas kingbird, $p \bar{e}$ kwēe.

Tyrannus vociferans Swains., Cassin's kingbird, pe'kwè.

Myiarclus mexicanus magister Ridgw., Arizona crested flycatcher, w̌$k \breve{o} \breve{o}^{\prime} c h \bar{e}-\bar{e}$.

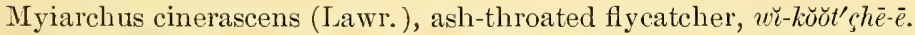

Sayornis saya (Bonap.), Say's Phœebe, pée'kwè.

Sayornis nigricans (Swains.), black Phœbe, putst'tu-ěh .

Contopus borealis (Swains.), olive-sided flycatcher, putst'tu-̌̌h .

Contopus richardsonii (Swains.), western wood pewee, çhe'é

Empidonax difficilis Baird, western flycatcher,

$\left.\begin{array}{l}\text { Empidonax traillii (Aud.), Traill's flycatcher, } \\ \text { Empidonax hammondi (Xantus), Hammond's flycatcher, }\end{array}\right\}$ chée-kach'té.

Empidonax wrightii Baird, Wright's flycatcher,

Pyrocephalus rubineus mexicanus (Scl.), vermilion flycatcher, tewy-w̌ǒts' $\overline{\text { }}$.

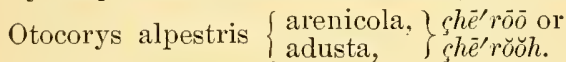

Pica pica hudsonica (Sab.), American magpie, pōe-sh $\bar{u}^{\prime} w \breve{\iota} h$. Ongwischey says they are common about the Moki villages.

Cyanocitta stelleri macrolopha (Baird), long-crested jay, lä-coich'cähn-ă. 
Aphelocoma woodhouseii (Baird), Woodhouse's jay, shou' ă-y̆̌h.

A phelocoma sieberii arizonæ Ridgw., Arizona jay, $\ddot{a} h-\ddot{a} h h^{\prime}$.

Corvus corax sinuatus (Wagl.), American raven, ŏng-ǔ̌sçh'́ey.

Corvus cryptoleucus Couch, white-necked raren, öng-w̌sçh'êy. "Two kinds; one name."

Corvus americanus Aud., American crow, ŏng-w̌sçh'hö-yŭh.

Nucifraga columbiana (Wils.), Clarke's nutcracker, yäh-päh (= accent).

Cyanocephalus cyanocephalus (Wied), piñon jay, $\ddot{a} h^{\prime} \ddot{a} h$.

Molothrus ater obscurus (Gmel.), dwarf cowbird, käh-nĕch' tō-ǩŏŏtsk'kă.

Xanthocephalus xanthocephalus (Bonap.), yellow-headed blackbird, tō$k \breve{o} \breve{t s k} k^{\prime} k$. This is a generic name for blackbirds.

Agelaius phœniceus longirostris (Salvad.), Sonoran red-wing, päh-lä'-

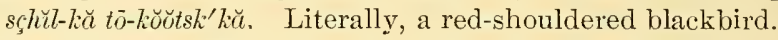

Sturnella magna neglecta (Aud.), wester'n meadowlark, puw-çhēle'low-ă.

Icterus parisorum Bonap., Scott's oriole, öh'mä-tow-ă-mŏn-ō.

Icterus cucullatus nelsoni Ridgw., Arizona hooded oriole, mōw'tow-ămŏn-ō.

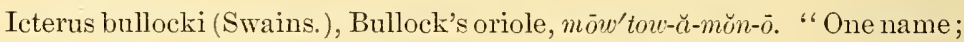
two kinds."

Scolecophagus cyanocephalus (Wagl.), Brewer's blackbird, tō-Ǩŏŏtsk'kă.

Coccothraustes vespertinus montanus (Ridgw.), western evening grosbeak, $t s \bar{e}-k \breve{a} c h t^{\prime} t \bar{e}$.

Carpodacus purpureus californicus Baird, California purple finch, till-hua'

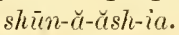

Carpodacus cassini Baird, Cassin's purple finch, tül-hua' shün-ă-ăsh-ia. "One name; two kinds."

Carpodacus mexicanus frontalis (Say), house finch, sçhē-pīle' shīn-ă-ăsh-ia Loxia curvirostra stricklandi Ridgw., Mexican crossbill, hä-rōōm'şhü-wŭh .

Leucosticte australis Ridgw, brown-capped leucosticte. "He no live Moki country" (which is true).

Spinis tristis pallidus Mearns, western goldfinch, sçhē-hûrp'chēe-e.

Spinus psaltria (Say), Arkansas goldfinch (var. arizonæincluded), tsée-kä $h^{\prime}$ ç̧ $\bar{e}-\bar{e}$ or coim'mŏp-mi-air-zhō.

Spinus pinus (Wils.), pine siskin, pou-coupt'çhe-e-e.

Calcarius ornatus (Towns.), chestnut-collared longspur, päh-lä'çlè-rø̌ŏh.

Rhynchophanes maccownii (Lawr.), MIcCown's longspur, coim'ŏp-çherǒ̌h

Poocæetes gramineus confinis Baird, western vesper sparrow, päh-lä'shŭ-cä.

Ammodramus sandwichensis alaudinus (Bonap.), western savanna sparrow, shè-huap' çhè-e.

Ammodramus savannarum perpallidus (Coues), western grasshopper spar-

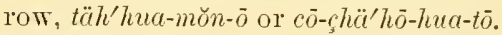

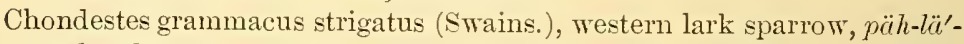
trlll-cäh-pbe.

Zonotrichia leucophrys (Forst.), white-crowned sparrow, cō-ch $\ddot{a} \prime g \bar{o}-p \ddot{a}$ şhēe- 
Zonotrichia leucophrys intermedia Ridgw., intermediate sparrow, cō-chliä'gō-pä çhēe-e.

Spizella socialis arizonæ Coues, western chipping sparrow, päh-läág $\bar{o}-p \ddot{a}$.

Spizella pallida (Swains.), clay-colored sparrow, pu-llü̈m'çhu-wŭh .

Spizella breweri Cass., Brewer's sparrow, pu-lhäm'ç' hu-wйh.

Spizella atrigularis (Cab.), black-chinned sparrow, coim'žp--le-e-que .

Junco hyemalis (Linn.), slate-colored junco, ne--wy'tōke-çhu. They apply

the same name to the other juncos, J. hyemalis shufeldti, J. annectens, $J$. caniceps, and $J$. phronotus dorsalis.

A mpinispiza bilineata (Cass.), black-throated sparrow, dŭtope'chēe-e.

Amphispiza belli nevadensis (Ridgw.), sage sparrow, çhierz-y̌̆-ē-kgōōt.

Peucæa cassini (Woodli.), Cassin's sparrow, pung'tō-qua.

Peucæa ruficeps boucardi (Scl.), Boucard's sparrow, pıng'tō-qua.

Melospiza fasciata $\left\{\begin{array}{l}\text { fallax, } \\ \text { montana, }\end{array}\right\} h \bar{e}^{\prime} \bar{o}-\check{\text { th }} \mathrm{c} h-\bar{e}$.

Melospiza lincolnii (Aud.), Lincolı's sparrow, päh-lä’hō-māyl-lŭ.

Pipilo maculatus megalonyx (Baird), spurred towhee, coim'bwē-qua.

Pipilo chlorurus (Towns.), green-tailed towhee, cō-chä'bwè-qua.

Pipilo fuscus mesoleucus (Baird), cañon to whee, $p y-y i r t^{\prime} t s \bar{e}-\bar{e}$.

Pipilo aberti Baird, Abert's towhee. Same as above. "One name; two kinds."

Cardinalis cardinalis superbus Ridgw., Arizona cardinal, päh-lä'lō-kw̌tskkŏn. Red $\left(p \ddot{a} h^{\prime} l \ddot{a}\right)$ is the favorite color with Mokis, as with other Indians, and any bright mark of that color, however small, serves as a specific character in their nomenclature; even birds of reddish or rufous color are often designated by them as "red birds." They were very enthusiastic in their expression of admiration when shown a mounted specimen of the vermilion flycatcher, and when Ongwischey caught sight of a male cardinal that some friend had sent to me from Agua Fria he begged so earnestly for me to stuff it for him that I did so, as it was the only time that he ever asked for anything that he saw in my house, or permitted the other Mokis to do so.

Pyrrhuloxia sinuata beckhami Ridgw., Arizona pyrrhuloxia, päh-lä'lókw̌̆tsk-kĭn.

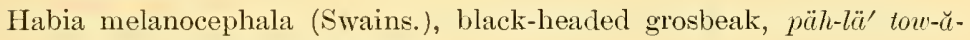

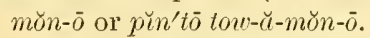

Guiraca cærulea eurhyncha Coues, western blue grosbeak, päh $h$-lä'jōōorōōo.

Passerina amœena (Say), lazuli bunting, çhä-quð̌ck'tä.

Spiza americana (Gmel.), dickcissel, sçhēe-pŏct'çhēe-è .

Calamospiza melanocorys Stejn., lark bunting, kēë-üh'rōō.

Piranga ludoviciana (Wils.), Louisiana tanager, mōw' tow-č-mŏn-̄̄.

Piranga hepatica Swains., hepatic tanager, $\ddot{a} h-\ddot{a} h ' s c ̧ h \bar{e}-\ddot{a} h$.

Piranga rubra cooperi Ridgw., Cooper's tanager, teu-ē-buăt'sē or päh-lä' tow- ̌̆-mŏn-ō.

Progne subis (Linn.), purple martin, shä-qua wow-wow'ŭh-k'ia.

Petrochelidon lunifrons (Say), cliff swallow, ō-wow' wow-wow' ̌̌ h-k'iu. 
Chelidon erythrogastra (Bodd.), barn swallow, çhö-cow wow-wow' ŭh-k'ia. Tachycineta thalassina (Swains.), violet-green swallow, wow-uov' $\breve{u} h-k^{\prime} i a$. Clivicola riparia (Linn.), bank swallow, chûr-hârth' wow-wow' üh-k'ia.

Stelgidopteryx serripennis (Aud.), rough-winged swallow, chûr-hûrbp' wow-wow' $\breve{\iota} h-k$ 'ia. This and the above are "river swallows."

Phainopepla nitens (Swains.), phainopepla, lä-kowtz'kälnn-nŭ.

Lanius ludovicianus excubitorides (Swains.), white-rumped shrike, $m \bar{o}^{\prime}$ çhॅn-e.e.

Vireo gilvus (Vieill.), warbling vireo, çhè-hîrp'çhēe-e. The same name was applied to the following species: V. solitarius, T. solitarius plumbeus, $V$. bellii pusillus, and $\mathrm{V}$. vicinior.

Helminthophila luciæ (Cooper), Lucy's warbler, päh-lä'gō-pŭh.

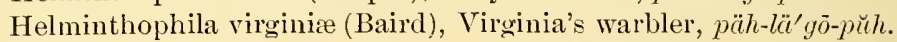

Helminthophila ruficapilla gutturalis Ridgw., Calaveras warbler, shume'$t s \bar{e}-k \ddot{a} \prime c h \bar{e}-k \ddot{a}-c, h \bar{e}$.

Helminthophila celata lutescens (Ridgw.), lutescent warbler. The same name as above.

Dendroica restiva (Gmel.), yellow warbler, päh'v̌n-shear-ž̆.

Dendroica auduboni (Towns.), Audubon's warbler, tshē-kă $k^{\prime} q u a-p \bar{e}$.

Dendroica graciæ Baird, Grace's warbler, çhēe-kăk'wë-pé.

Dendroica nigrescens (Towns.), black-throated gray warbler, tilll-whāy'hĕn-hûrk-ǩ̆.

Geothlypis macgillivrayi (Aud.), Macgillivray's warbler, mäsh'quc-pé.

Geothlypis trichas occidentalis Brewst., western yellow-throat, $m y-y e \bar{u}^{\prime} z \bar{o}$.

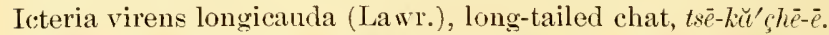

Sylvania pusilla pileolata (Pall.), pileolated warbler, $m y-y \bar{a}^{\prime} z \bar{o}$.

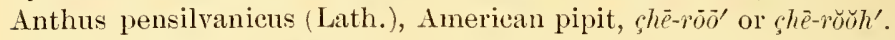

Cinclus mexicanus Swains., American dipper. This bird is unknown to the Mokis. There are no suitable places for it about their villages, as their water supply is derived from small springs or cisterns made for storing rain water, there being no mountain streams.

Oroscoptes montanus (Towns.), sage thrasher, mä-çhēte'çhè-è.

Mimus polyglottos (Linn.), mockingbird, coi-çhä'çhe-e-e. The first part of this name has reference to its pale or whitish colors.

Harporhynchus crissalis (Henry), crissal thrasher, will-pŏm'çho-wřh. The Mokis are unacquainted with the other Arizona thrashers.

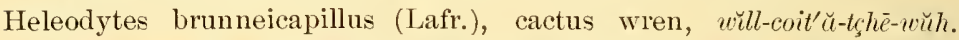
Ongwischey said that he visited San Carlos Indian agency, on the Gila river, seven years ago and there saw these large wrens among the tall cactuses.

Salpinctes obsoletus (Say), rock wren, pť̉lt'çhē-rwŭhl.

Catherpes mexicanus conspersus Ridgw., cañon wren, ptil'push-qua.

Thryothorus bewickii leucogaster Baird, Baird's wren, tult'çhē-wǔh .

Troglodytes aëdon aztecus Baird, western house wren, lill'push-qua ter'tŭck-ă. Singing wren.

Troglodytes hiemalis (pacificus Baird?), winter wren, ptıl'push-qua. 
Cistothorus palustris (Wils.), ${ }^{1}$ long-billed marsh wren, lï'çhy-ō.

Sitta carolinensis aculeata (Cass.), slender-billed nuthatch, höpe'yung-äh. Woodpecker!

Sitta pygniæa Vig., pygmy nuthatch, hōpe-yung'hoi-y̆̆.

Parus inornatus griseus Ridgw., gray titmouse, sè $k \bar{o} \bar{g} m-a ̈ h$.

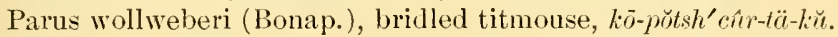

Parus gambeli Ridgw., mountain chickadee, kō-pussth'çhứ-lī-lŭ.

Psaltriparus plumbeus Baird, lead-colored bush-tit, were'were-you-mŭh.

Auriparus flaviceps (Sund.), verdin. Same name as the last.

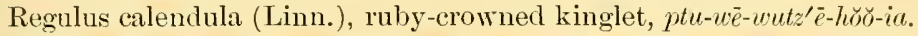

Polioptila cærulea obscura Ridgw., western gnatcatcher, shē-päy'bě h.

Polioptila plumbea Baird, plumbeous gnateatcher, sŭsh-shē'çhè-ēe.

Myadestes townsendii (Aud.), Townsend's solitaire, quē-quèl'mä̈çh-çhă.

Turdus ustulatus Nutt., russet-backed thrush, pin'tō çhēe-e. Spotted little bird.

Turdus aonalaschkæ Gmel., dwarf hermit thrush, p̌n'tō chēe-e. Var. auduboni of course received the same name.

Merula migratoria propinqua Ridgw., western robin, ptu-vēe-wîrtz'e

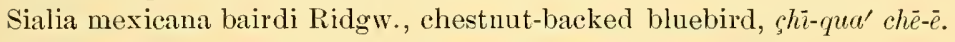

Sialia aretica Swains., mountain bluebird, çhö-rōō (= accent).

In the accompanying illustration the central figure represents Ong-wisch'-èy (raven); the others two young Moki huntersTäh-wy'-ōmb (stick) and Show'-yŭh (signifying bat, but also meaning sundown or twilight).

1 The long-billed marsh wren of Arizona has been called the subspecies palludicola of Baird; but as the type of C.p. palludicola came from the Pacific coast region, and represents a darker geographical form than that inhabiting Arizona-as I am informed by $\mathrm{Mr}$ Oberholser. who is engaged in making a eritical study of the marsh wrens-I have given only its specific name. 





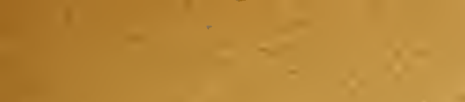

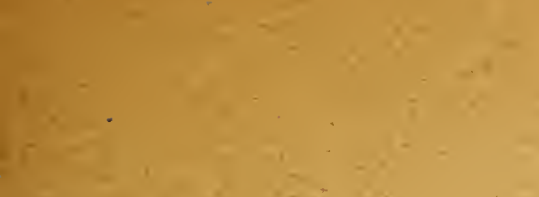

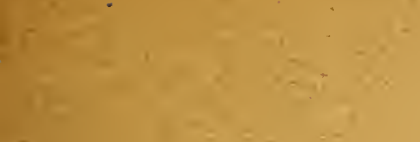

8

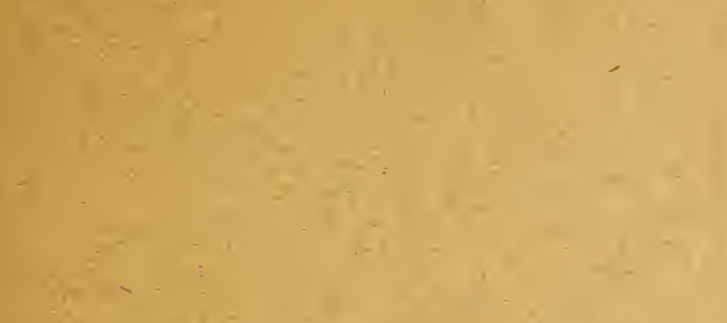

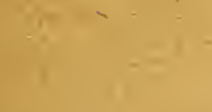

$$
\text { 1 }
$$

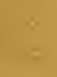$$
\cos ^{2+\infty}
$$

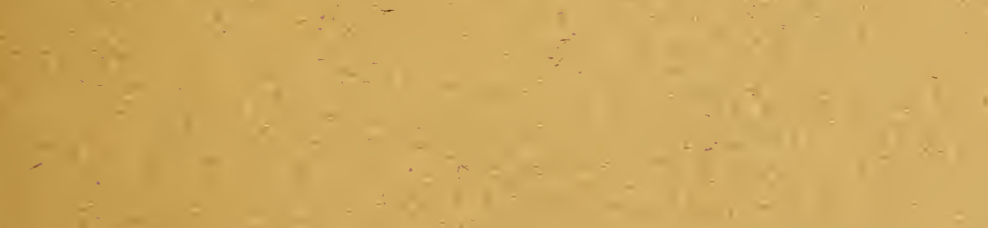$$
x-1=0=0
$$

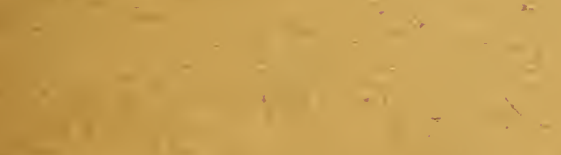




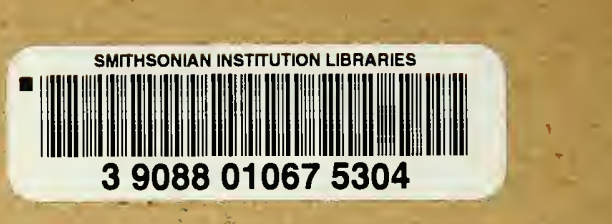

SMTHSONIAN INSTTUUTION LIBRARIES

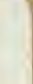

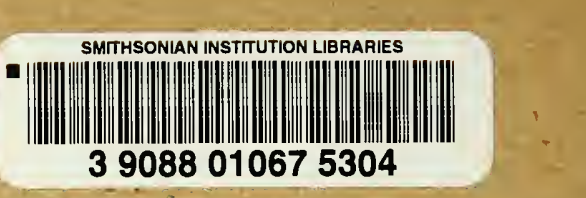

$\checkmark$

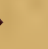

$\therefore$

$\because$

$x^{\prime}$

$\therefore-\cdots$

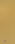

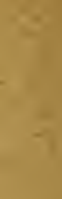

( 